$\begin{array}{llr}\text { Universitas } & \text { Vol. } 37 \\ \text { Núm. } 78\end{array}$

Artículos

\title{
Medición de la capa híbrida resultante del uso de cementos autograbadores de uno y dos pasos*
}

\author{
Hybrid Layer Measurement after Using One-Step and Two-Step Self-Etching Cements
}

Ligia Johanna Vásquez Dominguez

Práctica privada, Colombia

ORCID: http://orcid.org/0000-0002-1042-7580

Guillermo Arreola Martínez

Práctica privada, México

ORCID: http://orcid.org/0000-0003-4678-4739

Jaime Larriva Loyola

Práctica privada, Ecuador

ORCID: http://orcid.org/0000-0003-0555-3571

Adriana Rodríguez Ciódaro

Pontificia Universidad Javeriana, Colombia

ORCID: http://orcid.org/0000-0002-6640-3975

Edgar Humberto Güiza Cristancho ${ }^{\text {a }}$

Pontificia Universidad Javeriana, Colombia

eguiza2010@yahoo.com

ORCID: http://orcid.org/0000-0001-6883-8094
DOI: https://doi.org/10.11144/Javeriana.uo37-78.mchr Redalyc: http://www.redalyc.org/articulo.oa? id $=231260072004$

Fecha de recepción: 25 Enero 2017 Fecha de aprobación: 16 Marzo 2018 Fecha de publicación: 18 Junio 2018

\section{Resumen:}

Antecedentes: La formación de la capa híbrida debe ser proporcional a la capacidad de los cementos para retener con éxito postes de fibra de vidrio en el interior del conducto preparado endodónticamente. Objetivos: Medir el espesor de la capa híbrida en los tercios coronal y apical después de usar cementos autograbadores de uno y dos pasos. Métodos: Se realizó un estudio experimental con 30 premolares humanos que se trataron endodónticamente y se asignaron a tres grupos. Se cementaron postes de fibra de vidrio con tres cementos siguiendo las instrucciones de los fabricantes. Se realizaron cortes coronales y apicales y se cuantificó el grosor de la capa híbrida por microscopía electrónica de barrido. Los datos se analizaron con las pruebas estadísticas $\mathrm{H}$ de Kruskal-Wallis y U de Mann-Whitney ( $<$ 0,05). Resultados: El promedio del grosor de la capa híbrida fue de 0,74 mm con Multilink $\mathrm{N}^{\circ}$ (grupo 1), 0,24 mm con RelyX Ultimate (grupo 2) y 0,03 mm con RelyX U200 (grupo 3) ( coronal y apical fueron: $1,29 \mathrm{~mm}$ y $0,19 \mathrm{~mm}$ para el grupo $1 ; 0,33 \mathrm{~mm}$ y $0,14 \mathrm{~mm}$ para el grupo 2 y $0,26 \mathrm{~mm}$ y $0,23 \mathrm{~mm}$ para el grupo 3, diferencias que fueron estadísticamente significativas $(\mathrm{p}=0,043)$. Conclusiones: El espesor de la capa híbrida formada con el uso de los cementos de dos pasos fue mayor que con el cemento de un solo paso, especialmente en la porción coronal.

Palabras clave: biomateriales, capa híbrida, cemento autograbador, cemento autograbador de dos pasos, cemento autograbador de un paso, interfase, materiales dentales, odontología, poste de fibra de vidrio, prostodoncia, rehabilitación oral.

\begin{abstract}
:
Background: Hybrid layer formation should be proportional to cement properties to successfully retain fiberglass posts into root canals. Purpose: To measure of hybrid layer thickness in coronal and apical thirds after using one-step and two-step self-etching cements. Methods: This experimental study used 30 human premolars with root-canal treatments, which were assigned into three groups. Fiberglass posts were cemented with three types of self-etching cements following manufacturers' instructions. Roots were cut in the coronal and apical thirds and hybrid layer thickness was quantified through scanning electron microscopy. Data were analyzed with Kruskal-Wallis and Mann-Whitney tests $(\mathrm{p}<0.05)$. Results: Average thickness of the hybrid layer was $0.74 \mu \mathrm{m}$ with Multilink $\mathrm{N}^{\mathrm{rx}}$ (group 1), $0.24 \mu \mathrm{m}$ with RelyX Ultimate ${ }^{\mathrm{mx}}$ (group 2), and $0.03 \mu \mathrm{m}$ with RelyX U200 $0^{\mathrm{ru}}$ (group 3) (p=0.0092).
\end{abstract}

Notas de autor:

$$
\text { a Autor de correspondencia. Correo electrónico: eguiza2010@yahoo.com }
$$


Average thickness in coronal and apical thirds were: $1.29 \mu \mathrm{m}$ and $0.19 \mu \mathrm{m}$ for group 1; $0.33 \mu \mathrm{m}$ and $0.14 \mu \mathrm{m}$ for group 2; and $0.26 \mu \mathrm{m}$ and $0.23 \mu \mathrm{m}$ for group 3 , differences that were statistically significant $(\mathrm{p}=0.043)$. Conclusions: The thickness of the hybrid layer formed after suing two-step cements was greater than that of one-step cement, especially in the tooth coronal third. Keywords: fiber-glass post, hybrid layer, interface, one-step self-etching cement, self-etching cement, two-step self-etching cement.

\section{Introducción}

Por la pérdida de tejido vital, los dientes tratados endodónticamente presentan modificaciones en las propiedades biomecánicas como alteración en las fibras colágenas, disminución de la humedad y pérdida de la estructura dentaria (1). Esto aumenta la probabilidad de fractura con cualquier tipo de restauración (2).

Durante años se han investigado diferentes formas de rehabilitar dientes tratados endodónticamente con el uso de núcleos colados (aleaciones metálicas como oro tipo III y metal base) (3) y postes prefabricados principalmente en titanio y fibra de vidrio. Las ventajas de los postes de fibra de vidrio frente a las aleaciones metálicas son una mayor estética por ser de un color similar al diente, fácil manipulación y bajo costo $(4,5)$. Además, en los postes metálicos, por su alta rigidez y módulo elástico mayor que el de la dentina, las fuerzas se dirigen a puntos críticos impredecibles, lo que ocasiona fracturas longitudinales de la raíz y, en consecuencia, la pérdida del diente (6). Por otra parte, la fibra de vidrio presenta un módulo elástico cercano al de la dentina (7).

Para las restauraciones con postes de fibra de vidrio se utiliza la técnica de cementación adhesiva (8). La capacidad de los diferentes cementos para retener con éxito postes de fibra de vidrio en el interior del conducto está relacionada con las propiedades mecánicas del cemento, su eficacia adhesiva ante las dos superficies con las que entra en contacto y durabilidad, la configuración del poste y la preparación del conducto. Estos factores influyen en la distribución de la fuerza sobre la capa de cemento, lo que aumenta el riesgo de fractura (9). Los principios de unión están basados en la penetración del cemento dentro de los túbulos destinarios y la formación de las líneas de resina, por la interacción con los elementos orgánicos de la dentina peritubular desmineralizada (10).

Los cementos de grabado total se han usado, ya que su mecanismo de acción es disolver el barrillo dentinario y acondicionar su capa superficial. Así, se remueve gran parte de los elementos inorgánicos, se expone la malla colágena y aumenta la permeabilidad de los túbulos dentinales que serán infiltrados para formar las líneas de resina y la capa híbrida (11). Estos se han remplazado por los cementos autograbadores de séptima generación, cuya técnica se ha simplificado por tener en una misma solución o frasco los monómeros acídicos hidrofílicos, solventes orgánicos y agua (12).

Los nuevos materiales usados en la cementación adhesiva se prueban mediante procedimientos de resistencia a la fractura, que está directamente relacionada con el espesor de la capa híbrida formada entre el cemento y la dentina. Se ha establecido que, a mayor profundidad de las líneas de cemento resinoso, mayor es la resistencia al desalojo del poste (13). Mejorar la calidad de la unión poste-cemento-dentina es uno de los objetivos básicos para aumentar el éxito de las restauraciones en dientes tratados endodónticamente (14).

El cemento es una sustancia sólida cuyo objetivo es unir dos superficies. En odontología, actualmente existen tres tipos de cementos: no adhesivos (fosfato de zinc), de unión química (policarboxilato, ionómero de vidrio e ionómero de vidrio modificado con resina) y de adhesión micromecánica (cementos con base en dimetacrilato), como los cementos autograbadores (15). La mayoría de los cementos de resina necesita un pretratamiento del sustrato dental para promover la adhesión a los tejidos dentarios. Este pretratamiento puede realizarse mediante grabado total con ácido fosfórico, un imprimador y un adhesivo, pero recientemente se han introducido cementos autograbadores que evitan el tradicional grabado total y sus múltiples pasos, y con ello se ha logrado la cementación en un solo paso (6). 
Con el avance tecnológico, las casas comerciales han buscado disminuir los pasos clínicos y facilitar el uso de los cementos, evitar las posibles fallas técnicas en el momento de la preparación del sustrato y disminuir el tiempo de trabajo del odontólogo (16). Para obtener resultados óptimos, los postes deben tener propiedades físicas similares a la dentina, ser biocompatibles en el ambiente oral y actuar como amortiguadores de fuerzas para disminuir la tensión dirigida hacia la estructura dental residual (16). Los postes de fibra de vidrio son químicamente compatibles con bisfenoglicidilmetacrilato (Bis-GMA), material contenido en los cementos resinosos $(8,9)$. Por lo tanto, el uso de una técnica de cementación adhesiva reducirá el estrés creado por el poste en el interior del conducto (10).

Bitter y colaboradores (12) concluyeron que no hubo diferencias significativas en la interfase cementodentina cuando compararon un sistema autograbador con un sistema de grabado total. Como resultado de su investigación, mostraron una capa híbrida homogénea dentro del conducto radicular para ambos sistemas de cementos. Sridhara y colaboradores (13) compararon el espesor de la capa híbrida entre dos tipos de cementos: Panavia $F^{\oplus}$ (autoadhesivo) y All Bond $2^{\circ}$ (grabado total). Concluyeron que con All Bond 2 el espesor de la capa híbrida es mejor y más efectivo, ya que crea entrelazados micromecánicos entre el material adhesivo y la dentina grabada. Por otra parte, Esclassan Noirrit y colaboradores (17) compararon las técnicas de autograbado y grabado total en cuanto a la formación de capa híbrida. No hallaron diferencias significativas entre estas dos técnicas.

Teniendo en cuenta el avance en la investigación de materiales más eficientes y la ausencia de reportes que muestren el espesor de la capa híbrida con el uso de cementos autograbadores de un paso (RelyX U200 ) y dos pasos (Multilink $\mathrm{N}^{\bullet}$ y RelyX Ultimate ${ }^{\odot}$ ), se planteó la siguiente pregunta: ¿̨cuál es el espesor de la capa híbrida en los tercios coronal (línea amelocementaria) y apical (a $5 \mathrm{~mm}$ del ápice) al usar cementos autograbadores de un paso (RelyX U200 ${ }^{\circ}$ y dos pasos (Multilink $\mathrm{N}^{\bullet}$ y RelyX Ultimate ${ }^{\circ}$ ) a través de microscopía electrónica de barrido (MEB)?

\section{Materiales y métodos}

Previo aval del Comité de Investigación y Ética de la Facultad de Odontología de la Pontificia Universidad Javeriana de Bogotá, Colombia, se realizó un estudio experimental. Se usaron 30 premolares humanos superiores e inferiores, unirradiculares o multirradiculares, extraídos por indicación ortodóntica, previa firma del consentimiento de donación de órganos por parte de los pacientes. Se utilizaron dientes permanentes sin caries y sin tratamientos de conductos previos. Se excluyeron dientes con fractura radicular y defectos de cierre apical como falta de formación o reabsorción interna. Los dientes se desinfectaron con cloramina $\mathrm{T}$ al 0,5\%, se sumergieron en agua destilada y se almacenaron a $4{ }^{\circ} \mathrm{C}$ hasta el momento de la preparación.

Para el tratamiento endodóntico se realizó el acceso convencional con una fresa redonda \# 4. Se tomó la conductometría ubicando radiográficamente el límite conducto-dentina-cemento. Con limas de níquel titanio (ProTaper ${ }^{\circ}$, Denstply, Maillefer) se ensanchó el conducto para terminar su preparación. Se irrigó con solución de hipoclorito de sodio al 5,25 \% y, finalmente, con suero fisiológico. La obturación del conducto se realizó con conos de gutapercha (ProTaper, Denstply, Maillefer) y cemento sellador (Sealapex ${ }^{\oplus}$, Kerr Hawe) por medio de la técnica de condensación lateral. Por último, se realizó el corte de penachos con un instrumento caliente.

A las 24 horas se desobturó el conducto con fresas de baja velocidad Gates Gliden \# 2 (Denstply, Maillefer) y se preparó con la fresa suministrada por el sistema de postes (Tenax Fiber White ${ }^{\curvearrowleft}$ Coltene). Se irrigó con una solución de hipoclorito de sodio al 5,25 \% y se secó con puntas de papel. Se distribuyó la muestra aleatoriamente en tres grupos de 10 dientes y se cementaron los postes de fibra de vidrio (Tenax Fiber White, Coltene) así: el grupo 1 se cementó con Multilink $\mathrm{N}^{\bullet}$ (Ivoclar, Vivadent); el grupo 2, con RelyX Ultimate (3M, ESPE), y el grupo 3, con RelyX U200 (3M, ESPE). 
Para la cementación con Multilink $\mathrm{N}^{\ominus}$, se mezcló el contenido de los dos frascos imprimador $\mathrm{A} / \mathrm{B}$ en proporción 1:1, se llevó al conducto frotando por 15 segundos y se aireó. Acto seguido, se llevó el cemento al conducto por medio de la punta de automezcla, se introdujo el poste de fibra de vidrio en el conducto haciendo una ligera presión por 60 segundos y se fotopolimerizó durante 40 segundos con lámpara de fotocurado (Bluephase ${ }^{\oplus}$, Ivoclar Vivadent).

El procedimiento para aplicar el cemento RelyX Ultimate ${ }^{\bullet}$ fue el siguiente: se llevó el adhesivo autograbador (Scotchbond Universal ${ }^{\circ}, 3 \mathrm{M} \mathrm{ESPE}$ ) al interior del conducto, se aireó, se fotopolimerizó durante 20 segundos (Bluephase ${ }^{\bullet}$, Ivoclar Vivadent) y se aplicó el cemento dentro del conducto con una punta de automezcla. Se introdujo el poste de fibra de vidrio con ligera presión y se fotopolimerizó durante 40 segundos con lámpara de fotocurado (Bluephase $e^{\oplus}$ Ivoclar Vivadent).

El cemento RelyX U200 se aplicó directamente en el conducto. Se introdujo el poste de fibra de vidrio al conducto haciendo una ligera presión durante 60 segundos y se fotopolimerizó durante 40 segundos con una lámpara de fotocurado (Bluephase ${ }^{\circ}$ Ivoclar Vivadent).

Luego de la cementación del poste, se fijó cada diente en resina epóxica utilizando cilindros metálicos de $20 \mathrm{~mm}$ de diámetro por $25 \mathrm{~mm}$ de altura, hasta la unión amelocementaria. Se sumergió nuevamente en agua destilada a $4{ }^{\circ} \mathrm{C}$. A las 24 horas, los dientes se sometieron a un proceso de termociclado de 3000 ciclos (30 segundos a $5^{\circ} \mathrm{C}, 15$ segundos a temperatura ambiente y 30 segundos a $55^{\circ} \mathrm{C}$ ).

Se hicieron dos cortes transversales con disco diamantado (IsoMet Wafering Blades ${ }^{\circledR} 15 \mathrm{LC}$ ) con irrigación, a la altura de la línea amelocementaria (coronal) y a $5 \mathrm{~mm}$ del ápice (apical). Una vez secos los cortes, se recubrieron con grafito en intervalos de 4 segundos con 5 pulsos a una corriente de 50 amperios para un espesor de $50 \mathrm{~nm}$ (Quórum QR150R ). Con una MEB (Quanta $200 \mathrm{FEI}^{\circ}$ ) se tomaron imágenes con el detector de electrones secundarios y retrodispersados a una magnitud de hasta 6000X. Las imágenes se digitalizaron y se cuantificó el grosor en micras de la capa híbrida en los tercios coronal y apical descritos anteriormente, usando el programa Image $\mathrm{J}^{\circ}$.

Los valores obtenidos se tabularon y analizaron con las pruebas $\mathrm{H}$ de Kruskal-Wallis y U de Mann-Whitney para comparar los grupos ( confianza $=95 \%)$.

\section{Resultados}

Teniendo en cuenta que la medición se realizó con un programa de computador, se incluyeron para el análisis las imágenes que permitieron identificar la formación de la capa híbrida (figura 1) o las brechas (figura 2), tanto para los cortes coronales como para los apicales. Por esta razón, del total de 60 especímenes obtenidos, 6 no se pudieron analizar por distorsión de las imágenes al momento de la cuantificación. Se muestran los resultados de 18 muestras del grupo 1 (Multilink $\mathrm{N}^{\circ}$ ) (9 coronal y 9 apical), 19 muestras del grupo 2 (RelyX Ultimate $\left.^{\circ}\right)$ (10 coronal y 9 apical) y 17 muestras del grupo 3 (RelyX U200 $)(9$ coronal y 8 apical). 


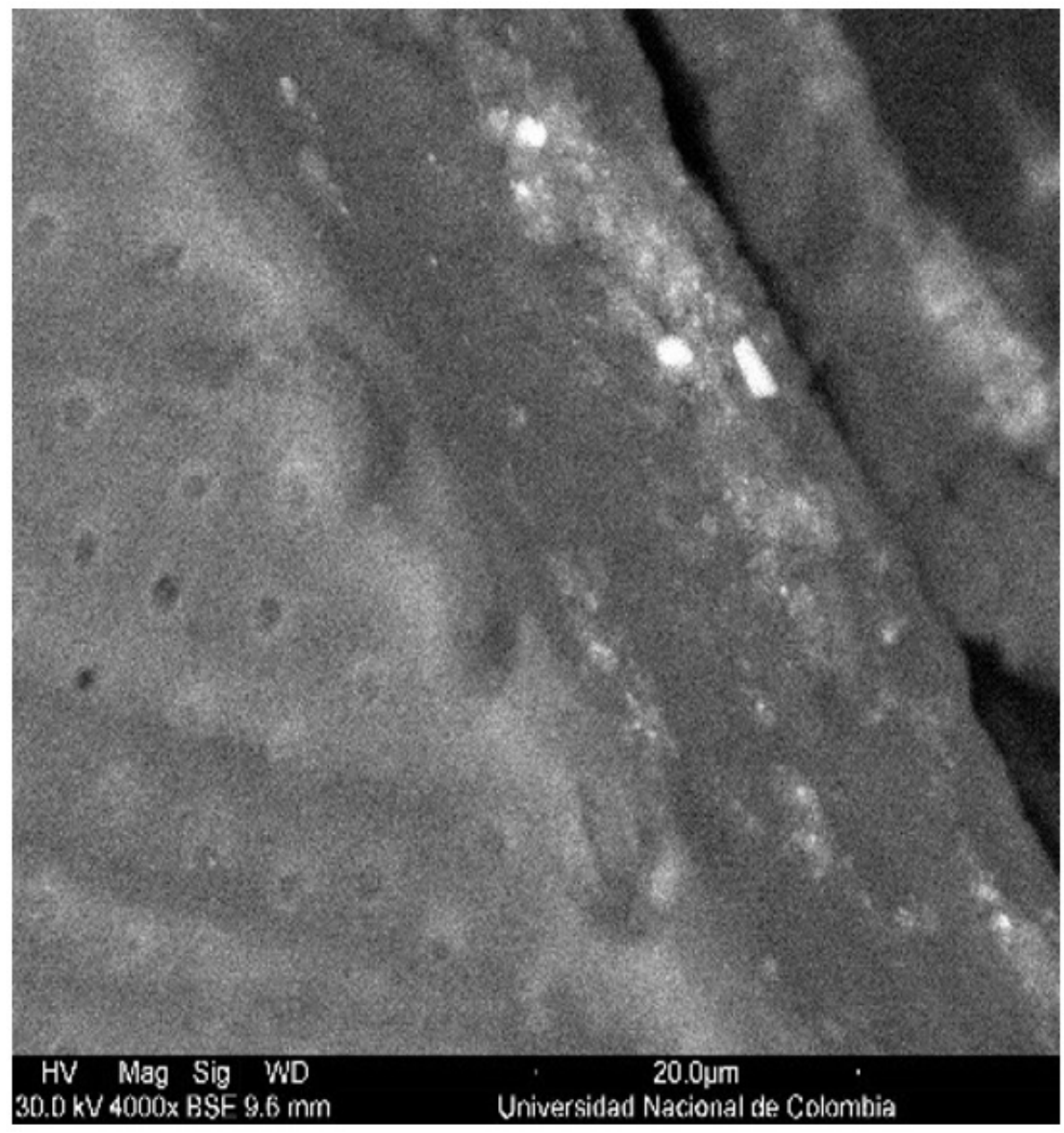

FIGURA 1

Capa híbrida formada en la porción coronal en un espécimen del grupo 1 Fuente: elaboración propia. 


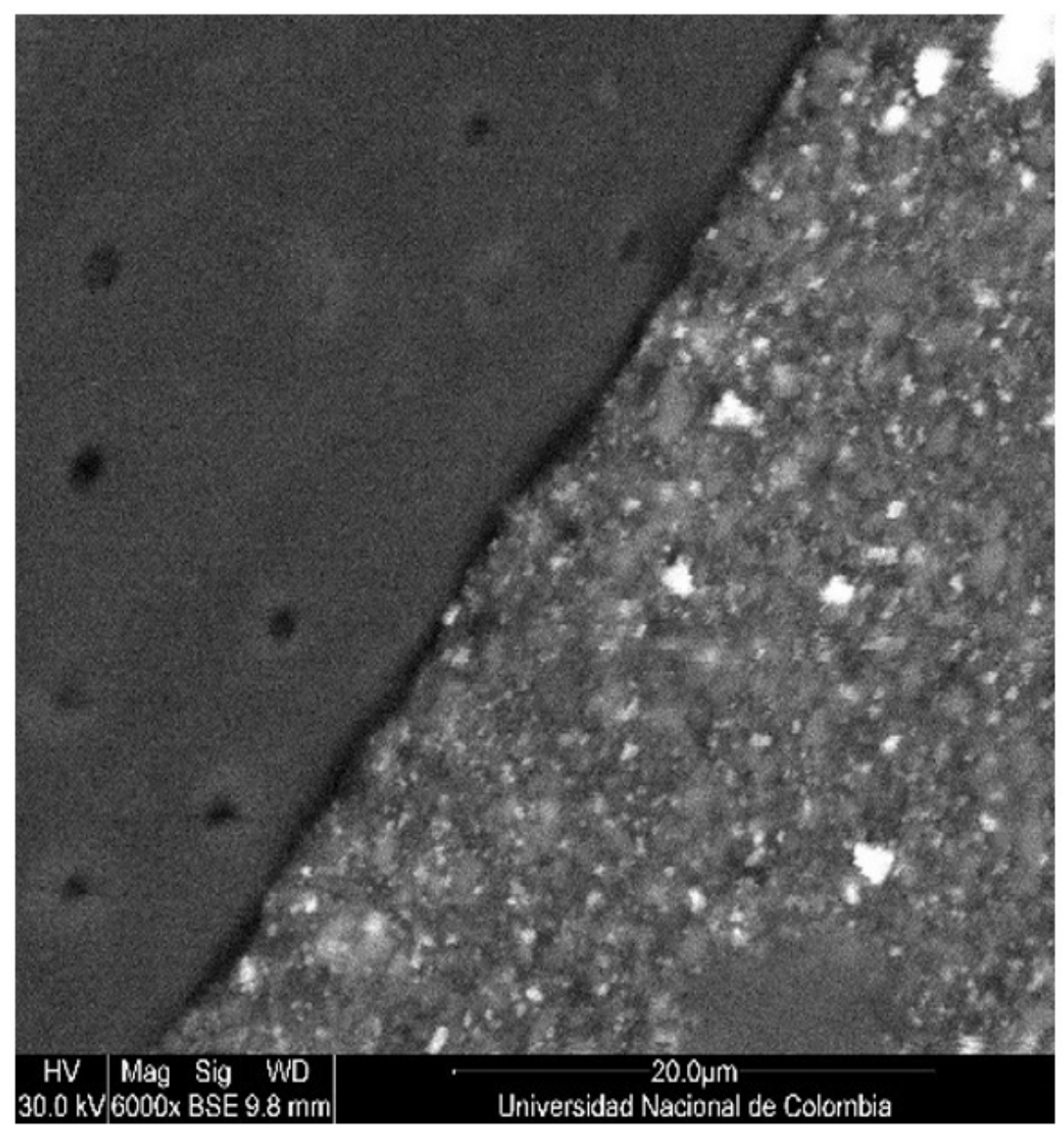

FIGURA 2

Ausencia de formación de la capa híbrida en la porción coronal en un espécimen del grupo 1 Fuente: elaboración propia.

El grupo 2 mostró mayor número de especímenes con capa híbrida formada, tanto en la porción coronal como en la apical, seguido por el grupo 1 (tabla 1 ).

TABLA 1

Comportamiento de las muestras según la formación de la capa híbrida por cada tipo de cemento y ubicación

\begin{tabular}{lcccc}
\hline \multirow{2}{*}{ Cemento } & \multicolumn{2}{c}{ Coronal } & \multicolumn{2}{c}{ Apical } \\
\cline { 2 - 5 } & Capa híbrida & Brecha & Capa híbrida & Brecha \\
\hline Grupo 1: Multilink N $^{\circ}$ & 8 & 1 & 7 & 2 \\
\hline Grupo 2: RelyX Ultimate $^{\circ}$ & 10 & 0 & 8 & 1 \\
\hline Grupo 3: RelyX U200 $^{\circ}$ & 8 & 1 & 4 & 4 \\
\hline
\end{tabular}

Fuente: elaboración propia.

Con respecto al grosor de la capa híbrida, se encontró que el cemento Multilink $\mathrm{N}^{\circledR}$ tuvo un promedio mayor que los otros dos cementos $(\mathrm{p}=0,0092)$. El mismo comportamiento se observó para el grosor en la porción coronal. Sin embargo, en la porción apical el mayor promedio se halló en el cemento RelyX U200, aunque esta diferencia no fue estadísticamente significativa. Las comparaciones entre los dos tercios muestran 
que para los grupos 1 y 2 los valores fueron significativamente más altos en la porción coronal que en la apical $(\mathrm{p}=0,046)($ tabla 2$)$.

TABLA 2

Promedio y desviación estándar en micras ( $\mathrm{mm})$ del grosor de la capa híbrida por tipo de cemento y ubicación

\begin{tabular}{lcccccc}
\hline \multirow{2}{*}{ Cemento } & \multicolumn{2}{c}{ Coronal } & \multicolumn{2}{c}{ Apical } & \multicolumn{2}{c}{ Total } \\
\cline { 2 - 7 } & Promedio & DE & Promedio & DE & Promedio & DE \\
\hline Multilink N $^{\circ}$ & 1,29 & 1,46 & 0,19 & 0,96 & 0,74 & 1,33 \\
\hline RelyX Ultimate $^{\circ}$ & 0,33 & 0,57 & 0,14 & 0,66 & 0,24 & 0,60 \\
\hline RelyX U200 $^{\circ}$ & 0,26 & 0,47 & 0,23 & 1,05 & 0,03 & 0,81 \\
\hline
\end{tabular}

Fuente: elaboración propia. DE: desviación estándar.

\section{Discusión}

La rehabilitación del diente tratado endodónticamente se dificulta por las características de las estructuras del diente cuando se pierde la pulpa dental. Una de las principales formas de rehabilitación en estos casos es el uso de retenedores intrarradiculares, cuyo éxito depende en parte de la fuerza de adhesión a la dentina producida por los cementos. Adicional a las pruebas de resistencia, el grosor de la capa híbrida puede utilizarse para establecer la efectividad de los materiales adhesivos usados en este sistema. La capa híbrida se define como la zona de interdifusión dentina-resina que se forma por la infiltración de monómeros de imprimador y adhesivo en la red de fibras colágenas expuestas, por la acción del acondicionador ácido sobre la dentina peri e intertubular, formada por la dentina radicular y el cemento de resina. El grosor de esta zona se relaciona con la resistencia y calidad de la restauración (3).

Existen diferentes tipos de cementos autograbadores según su $\mathrm{pH}$, los cuales se dividen en adhesivo de autograbado ultrasuave $(\mathrm{pH}>2,5)$, que presentan una capa híbrida de aproximadamente $300 \mathrm{~nm}$ de espesor; autograbador leve $(\mathrm{pH} \approx 2)$, cuya capa híbrida varía entre $0,5 \mu \mathrm{m}$ y $1 \mu \mathrm{m}$; y autograbador fuerte $(\mathrm{pH} \leq 1)$, que crea una capa híbrida totalmente desmineralizada y gruesa en la que las fibras de colágeno ya no están protegidas por la hidroxiapatita (18). En esta investigación se utilizaron los cementos autograbadores RelyX $\mathrm{U}-200^{\circ}$ (3M, ESPE) de un solo paso y grabado ultrasuave, cemento RelyX Ultimate ${ }^{\circ}$ (3M, ESPE) de dos pasos en el que se encuentran adhesivo y ácido en un solo frasco (Scotchbond ${ }^{\circ}$, Universal 3M, ESPE) de grabado ultrasuave y cemento Multilink $\mathrm{N}^{\oplus}$ (Ivoclar, Vivadent) que necesita imprimador (A y B) y es de grabado suave.

Además de la formación de la capa híbrida, también forman líneas de resina y zonas de interdifusión entre el cemento y la dentina, que se basan en la infiltración de resina en la superficie desmineralizada de la dentina $(19,20,21)$. Algunos estudios han mostrado que a mayor infiltración de la superficie desmineralizada de la dentina, mayor será la resistencia a la tracción (21).

En el presente estudio se observó la formación de capa híbrida superficial, pero no se evidenció la formación de líneas de resina, ni zonas de interdifusión dentina-resina. De acuerdo con Ferrari y colaboradores (21), la formación de las líneas de resina dependerá del grado de desmineralización de la dentina, lo que a su vez está relacionado con la permeabilidad aumentada de los túbulos dentinarios $(20,22)$.

Los cortes realizados en las muestras se hicieron en sentido transversal. Por lo tanto, se esperaba ver los conductos ubicados longitudinalmente, lo que no siempre ocurrió. Se observó que la orientación y dirección de los túbulos dentinarios no necesariamente van perpendicularmente al conducto. 
Los resultados obtenidos mostraron que la porción coronal de las muestras presentó mejor calidad de capa híbrida con respecto a la porción apical. Esto se podría explicar por la densidad o fluidez del cemento, la geometría del conducto radicular y la penetración de la luz, ya que esta disminuye de forma inversamente proporcional al cuadrado de la distancia (23). Esto concuerda con lo descrito por Lui (24), quien afirma que no se puede asegurar una completa polimerización de una resina cuando se introduce a una profundidad mayor a 4-5 $\mathrm{mm}$. También podría relacionarse con la capacidad del poste de fibra de vidrio para trasmitir la luz con una intensidad suficiente.

Los cementos autograbadores deben unirse de manera efectiva a la dentina y al poste para lograr una restauración más resistente y retentiva. En las imágenes de MEB se observa la unión íntima entre los cementos y el poste. Sin embargo, se identificaron espacios entre el cemento, los agentes adhesivos y la dentina. En algunos cortes apicales se encontró separación entre el cemento y la dentina, lo que coincide con los hallazgos de Bouillaguet y colaboradores (25), quienes describieron que la fuerza de contracción del medio de cementación en el interior del conducto radicular excede la fuerza de unión lograda entre la resina y la capa híbrida, lo que produce su separación.

En el presente estudio, siguiendo los protocolos de las casas comerciales, se utilizó hipoclorito como irrigador. Según Vilas y colaboradores (26), el uso de hipoclorito promueve cambios en el metabolismo celular por oxidación e inhibición enzimática. Además, los residuos del hipoclorito de sodio, al permanecer en el interior del túbulo dentinal, no permiten la polimerización completa de los monómeros de resina en la interfase adhesivo-resina.

El mecanismo de unión de los sistemas autograbadores se describe como una unión de enlaces dobles, los cuales se dividen en micromecánicos y de unión química (27). La unión micromecánica genera resistencia contra el esfuerzo mecánico y la unión química reduce la degradación hidrolítica. Los monómeros acídicos presentes en los sistemas autograbadores interactúan iónicamente con la hidroxiapatita y la malla colágena presente en el diente para formar una nanoestratificación, lo que mejora la calidad de la capa híbrida $(27,28)$.

Los cementos autograbadores de uno y dos pasos, al incorporar un ácido en su composición, no son lo suficientemente fuertes como para aumentar la permeabilidad de los túbulos dentinales y eliminar el barrillo dentinario. Por lo tanto, el barrillo dentinario se incorpora a su composición $(27,28)$, lo que conlleva la formación de una capa híbrida sin la presencia de las líneas de resina $(20,27)$. La formación de la capa híbrida va a influir en la retención con éxito del poste de fibra de vidrio en el interior del conducto, y cuanto mayor sea la profundidad del cemento resinoso, mayor será la resistencia del poste al desalojo.

\section{Conclusiones}

El espesor de la capa híbrida formada con el uso de los cementos de dos pasos fue mayor que con el cemento de un solo paso.

El número de especímenes con capa híbrida formada fue mayor con el uso de cementos de dos pasos.

La formación de capa híbrida, tanto en número como en grosor, fue mayor en la porción coronal que en la porción apical.

\section{Recomendaciones}

Diseñar un estudio en el que se compruebe la resistencia a la fractura con el uso de estos cementos. Evaluar el efecto del tiempo en la descementación de los postes al usar estos cementos. 


\section{Agradecimientos}

Al ingeniero Daniel Enrique Cañas, por su colaboración en el análisis estadístico.

\section{Referencias}

1. Preethi G, Kala M. Clinical evaluation of carbon fiber reinforced carbon endodontic post, glass fiber reinforced post with cast post and core: A one-year comparative clinical study. J Conserv Dent. 2008 Oct; 11(4): 162-7. https ://doi.org/10.4103/0972-0707.48841

2. Nakabayashi N, Nakamura M, Yasuda N. Hybrid layer as a dentin-bonding mechanism. J Esthet Dent. 1991 JulAug; 3(4): 133-8.

3. Morgano SM, Milot P. Clinical success of cast metal posts and cores. J Prosthet Dent. 1993 Jul; 70(1): 11-6.

4. Massa F, Dias C, Blos CE, Resistance to fracture of mandibular premolars restored using post-and-core systems. Quintessence Int. 2010 Jan; 41(1): 49-57.

5. Duarte S, Sartori N, Sadan A, Phark JH. Adhesive resin cements for bonding esthetic restorations: a review. Quintessence Dent Technol. 2011 Jan; 34: 40-66.

6. Nakabayashi N, Kojima K, Masuhara E. The promotion of adhesion by the infiltration of monomers into tooth substrates. J Biomed Mater Res. 1982 May; 16(3): 265-73.

7. Ferrari M, Vichi A, Grandini S. Efficacy of different adhesive techniques on bonding to root canal walls: an SEM investigation. Dent Mater. 2001 Sep; 17(5): 422-9.

8. Sahmali S, Demirel F, Saygili G. Comparison of in vitro tensile bond strengths of luting cements to metallic and tooth-colored posts. Int J Periodontics Restorative Dent. 2004 Nov; 24(3): 256-63.

9. Sorensen JA, Engelman MJ. Effect of post adaptation on fracture resistance of endodontically treated teeth.J Prosthet Dent. 1990 Oct; 64(4): 419-24.

10. Kugel G, Ferrari M. The science of bonding: from first to sixth generation. J Am Dent Assoc. 2000 Jun; 131 Suppl: 20S-25S.

11. Pashley DH, Tay FR. Aggressiveness of contemporary self-etching adhesives. Part II: etching effects on unground enamel. Dent Mater. 2001 Sep; 17(5): 430-44.

12. Bitter K, Gläser C, Neumann K, Blunck U, Frankenberger R. Analysis of resin-dentin interface morphology and bond strength evaluation of core materials for one stage post-endodontic restorations. PLoS One. 2014 Feb; 9(2): e86294. https://doi.org/10.1371/journal.pone.0086294

13. Sridhara KS, Mankar S, Jayshankar CM, Vinaya K. Scanning electron microscopic study of teeth restored with fiber posts and composite resin: An in vitro study. J Pharm Bioallied Sci. 2014 Jul; 6(Suppl 1): S74-S79.

14. Giachetti L, Bertini F, Scaminaci Russo D. Investigation into the nature of dentin resin tags: a scanning electron microscopic morphological analysis of demineralized bonded dentin. J Prosthet Dent. 2004 Sep; 92(3): 233-8.

15. Radovic I, Mazzitelli C, Chieffi N, Ferrari M. Evaluation of the adhesion of fiber posts cemented using different adhesive approaches. Eur J Oral Sci. 2008 Dec; 116(6): 557-63. https://doi.org/10.1111/j.1600-0722.2008.0 0577.x

16. Akgungor G, Akkayan B. Influence of dentin bonding agents and polymerization modes on the bond strength between translucent fiber posts and three dentin regions within a post space. J Prosthet Dent. 2006 May; 95(5): 368-78.

17. Esclassan Noirrit E, Grégoire G, Cournot M. Morphological study of fiber-reinforced post-bonding system-root dentin interface by evaluation of two bonding systems. J Dent. 2008 Mar; 36(3): 204-13. https://doi.org/10.1 016/j.jdent.2007.12.003

18. Koshiro K, Sidhu SK, Inoue S, Ikeda T, Sano H. New concept of resin-dentin interfacial adhesion: the nanointeraction zone. J Biomed Mater Res B Appl Biomater. 2006 May; 77(2): 401-8. 
19. Mannocci F, Innocenti M, Ferrari M, Watson TF. Confocal and scanning electron microscopic study of teeth restored with fiber posts, metal posts, and composite resins. J Endod. 1999 Dec; 25(12): 789-94.

20. Ozdemir E, Lin WS, Erkut S, Tuncer AH. Interfacial evaluation of endodontically treated teeth restored with selected adhesive materials and glass fiber posts: an SEM analysis. J Dent Sci. 2013 Dec; 8(4): 365-72. https:// doi.org/10.1016/j.jds.2012.03.021

21. Ferrari M, Vichi A, Grandini S, Goracci C. Efficacy of a self-curing adhesive-resin cement system on luting glassfiber posts into root canals: an SEM investigation. Int J Prosthodont. 2001 Nov-Dec; 14(6): 543-9.

22. da Silveira Teixeira C, Santos Felippe MC, Silva-Sousa YT, de Sousa-Neto MD. Interfacial evaluation of experimentally weakened roots restored with adhesive materials and fibre posts: an SEM analysis. J Dent. 2008 Sep; 36(9): 672-82. https://doi.org/10.1016/j.jdent.2008.04.020

23. Giannini M, Makishi P, Ayres AP, Vermelho PM, Fronza BM, Nikaido T, Tagami J. Self-etch adhesive systems: a literature review. Braz Dent J. 2015 Jan-Feb; 26(1): 3-10. https://doi.org/10.1590/0103-6440201302442

24. Lui JL. Depth of composite polymerization within simulated root canals using light-transmitting posts. Oper Dent. 1994; 19: 165-8.

25. Bouillaguet S, Troesch S, Wataha JC, Microtensile bond strength between adhesive cements and root canal dentin. Dent Mater. 2003 May;19(3): 199-205.

26. Vilas Boas Motta CA, Filadelfo Silva M, Wanderley Cruz JF, Carneiro Ribeirod F, Torres León BL. The influence of $1 \%$ sodium hypochlorite in the bond strength between the radicular dentin and the fiber post using three cementing agents. Rev Odonto Cienc. 2015; 30(4): 173-8. https://doi.org/10.15448/1980-6523.2015.3.16736

27. Tay FR, Pashley DH, Aggressiveness of contemporary self-etching systems. I: Depth of penetration beyond dentin smear layers. Dent Mater. 2001 Jul; 17(4): 296-308.

28. Koibuchi H, Yasuda N, Nakabayashi N. Bonding to dentin with a self-etching primer: the effect of smear layers. Dent Mater. 2001 Mar; 17(2): 122-6.

\section{Notas}

* Artículo de investigación.

\section{Licencia Creative Commons CC BY 4.0}

Cómo citar este artículo: Vásquez Domínguez LJ, Arreola Martínez G, Larriva Loyola J, Rodríguez Ciódaro A, Güiza Cristancho EH. Medición de la capa híbrida resultante del uso de cementos autograbadores de uno y dos pasos. Univ Odontol. 2018 ene-jun; 37(78). https://doi.org/10.11144/Javeriana.uo37-78.mchr 